\title{
Cylindrospermopsis raciborskii (Woloszynska) Seenayya \& Subba Raju (Cyanobacteria): variação semanal e relações com fatores ambientais em um reservatório eutrófico, São Paulo, SP, Brasil
}

\author{
ANDRÉA TUCCI ${ }^{1,2,3}$ e CÉLIA L. SANT'ANNA ${ }^{2}$
}

(recebido: 1 de fevereiro de 2002; aceito: 3 de janeiro de 2003)

\begin{abstract}
Cylindrospermopsis raciborskii (Woloszynska) Seenayya \& Subba Raju (Cyanobacteria): weekly variation and relation with environmental factors in an eutrophic lake, São Paulo, SP, Brazil). Blooms of Cylindrospermopsis raciborskii have become more and more frequent in Brazilian reservoirs because of its high competitiveness in tropical eutrophic systems. This species is able to produce very aggressive toxins which cause problems to public health and environmental hazards. Our objective was to follow up variations of $C$. raciborskii density and its relation with the environmental parameters. The studied lake is located in the south of São Paulo city $\left(23^{\circ} 39^{\prime}\right.$ S and $\left.46^{\circ} 37^{\prime} \mathrm{W}\right)$. It is an eutrophic system with frequent blooms of C. raciborskii. Samples were collected weekly in the deepest part of the lake, from September/97 to September/98. Temperature profile, mixing zone, euphotic zone, $\mathrm{pH}$, conductivity, turbidity, dissolved oxygen, alkalinity, nutrients, chlorophyll $a$, C. raciborskii density and total phytoplanktonic density were analysed. The ordination of abiotic and biotic data was carried out using Principal Component Analysis (PCA) and Canonical Correspondence Analysis (CCA). During November and December/97 the highest values of $C$. raciborskii densities were observed. From $12 / 10$ to $12 / 17$, the species contributed with more than $50 \%$ to total density. In that period the water column was thermally stratified, temperature and turbidity were high and water transparency decreased. The autumn/winter of 1998 was characterized by the absence of the studied species, by unstratified water column, low water level and temperature, high values of transparency (the highest values observed in the lake) and a great increase in biomass of the macrophyte Eichhornia crassipes (Mart.) Solms. All these relations are presently discussed.
\end{abstract}

Key words - Cyanobacteria, Cylindrospermopsis raciborskii, eutrophic reservoir, weekly variation

RESUMO - (Cylindrospermopsis raciborskii (Woloszynska) Seenayya \& Subba Raju (Cyanobacteria): variação semanal e relações com fatores ambientais em um reservatório eutrófico, São Paulo, SP, Brasil). Florações de Cylindrospermopsis raciborskii têm sido cada vez mais freqüentes em reservatórios brasileiros em virtude da sua alta competitividade em ambientes tropicais eutrofizados. Esta espécie é produtora de toxinas extremamente agressivas, as quais podem causar problemas de saúde pública e danos ao meio ambiente. Nosso objetivo foi acompanhar a variação temporal da densidade desta população relacionando-a com variáveis ambientais e detectando os possíveis fatores que interferem no seu desenvolvimento. O lago estudado localiza-se na zona sul do Município de São Paulo (2339' S e 46 $47^{\circ}$ ' W). É um corpo d'água eutrofizado e vem apresentando florações freqüentes de C. raciborskii. Amostras de água foram coletadas semanalmente, na parte mais profunda do lago, durante o período de setembro/97 a setembro/98. Foram analisados o perfil térmico, zona de mistura, transparência, zona eufótica, turbidez, $\mathrm{pH}$, alcalinidade, condutividade, oxigênio dissolvido, nutrientes, clorofila $a$, densidade da população e densidade total da comunidade fitoplanctônica. A ordenação dos dados abióticos e bióticos foi feita através da análise de componentes principais (PCA) e análise de correspondência canônica (CCA). Durante os meses de novembro e dezembro/97 foram registrados os mais altos valores de densidade de C. raciborskii. Entre 10/12 e $17 / 12$, a espécie contribuiu com $50 \%$ para a densidade total da comunidade. Nesse período foram registradas estratificações térmicas, elevados valores de temperatura da água e de turbidez e baixos valores de transparência da água. Os meses de outono e inverno de 1998 foram caracterizados pela ausência da espécie estudada, desestratificação térmica da coluna d'água, baixas temperaturas da água, altos valores de transparência (os maiores já registrados para o ambiente) e pelo aumento considerável dos bancos da macrófita aquática flutuante Eichhornia crassipes (Mart.) Solms. Tais interrelações são discutidas no presente trabalho.

Palavras-chave - Cyanobacteria, Cylindrospermopsis raciborskii, reservatório eutrófico, variação semanal

\section{Introdução}

Florações de Cylindrospermopsis raciborskii (Woloszynska) Seenayya \& Subba Raju têm sido cada

1. Universidade Estadual de Feira de Santana, Departamento de Ciências Biológicas, Laboratório de Ficologia, Módulo 1. Km 03 BR 116, Campus Universitário, 44031-460 Feira de Santana, BA, Brasil.

2. Instituto de Botânica, Seção de Ficologia, Caixa Postal 4005, 01061-970 São Paulo, SP, Brasil.

3._Autor para correspondência: tucci@uefs.br vez mais freqüentes em reservatórios brasileiros o que torna esta espécie um dos mais importantes componentes das comunidades fitoplanctônicas (Branco \& Senna 1991, 1994, 1996a, Branco \& Cavalcanti 1999, Bouvy et al. 1999, Bouvy et al. 2000, Huszar et al. 2000, Komárková et al. 1999, Padisák 1997). Sua alta competitividade em ambientes eutrofizados, aliada à sua capacidade de formar florações e produzir toxinas, fazem desta espécie uma das Cyanobacterias mais estudadas tanto do ponto de 
vista ecológico como de saúde pública. Duas das toxinas produzidas são a cilindrospermopsina, um alcalóide com ação no fígado e rins (Ohtani et al. 1992, Lagos et al. 1999) e a potente toxina paralisante do tipo PSP (Paralytic Shellfish Poisons), que age no sistema neuromuscular (Lagos et al. 1999). As florações e a produção de toxinas comprometem a qualidade da água para abastecimento, além de aumentarem consideravelmente os seus custos de tratamento (Carmichael 1996, Codd 2000).

Cylindrospermopsis raciborskii foi descrita originalmente para região tropical mas, atualmente, vem formando florações também em ambientes temperados. Isto demonstra sua alta capacidade de adaptação e vantagem competitiva o que possibilitou a sua rápida expansão em diferentes zonas do globo terrestre (Padisák 1997, Sant'Anna \& Azevedo 2000).

Para a taxonomia de C. raciborskii, são importantes os trabalhos de Jeeji-Bai et al. (1977), Horecká \& Komárek (1979) e Komárková (1998) que, além de descreverem e ilustrarem muito bem a espécie, analisaram a problemática nomenclatural desde sua proposição original, feita com base em material coletado em lagos da Indonésia e incluída no gênero Anabaena, até a sua reavaliação e inclusão em Cylindrospermopsis.

No Brasil, o primeiro relato da ocorrência de C. raciborskii foi feito por Palmer (1969) para o Lago Paranoá em Brasília, que na época identificou a população encontrada como Aphanizomenon flos-aquae (L.) Ralfs. Atualmente, sabe-se que se tratava de C. raciborskii (Branco \& Senna 1991). Apenas na década de 80 surgiram referências da ocorrência desta espécie para outras regiões do Brasil: Sant'Anna et al. (1988) para a represa de Serraria, SP e Torgan \& Garcia (1989) para a Lagoa dos Patos, RS. A grande expansão na distribuição de $C$. raciborskii deu-se a partir da década de 90, coincidindo com o aumento da eutrofização dos sistemas aquáticos nas mais diversas regiões do país (Sant'Anna \& Azevedo 2000). Há indícios de que o forte evento El Niño de 97-98 tenha também favorecido o desenvolvimento desta espécie em outras regiões do país (Bouvy et al. 1999, 2000).

A despeito dos impactos causados pela dominância de C. raciborskii em lagos eutróficos, são ainda raros os trabalhos brasileiros que tratam especificamente da ecologia deste organismo. Nesse sentido, merecem destaque os trabalhos de Branco \& Senna (1994, 1996a) que atribuíram a dominância de $C$. raciborskii no Lago Paranoá, DF, ao processo de eutrofização artificial decorrente de impactos antrópicos; Souza et al. (1998) que estudaram a dominância de C. raciborskii no Rio
Pequeno, braço da Represa Billings, SP; Bouvy et al . (1999, 2000) que estudaram a dinâmica de C. raciborskii em reservatórios da região do semi-árido do nordeste; Komárková et al. (1999) que analisaram a variabilidade morfológica de $C$. raciborskii na Lagoa do Peri, SC; e Huszar et al. (2000), que analisaram o fitoplâncton de oito sistemas brasileiros, dos quais cinco são dominados por Cylindrospermopsis.

Especificamente para o lago estudado (Lago das Garças), os trabalhos de Sant'Anna et al. (1997), Nogueira \& Ramirez (1998), Bicudo C. et al. (1999), Bicudo D. et al. (1999) e Mercante \& Tucci (1999) apresentam importantes informações sobre a comunidade fitoplanctônica em geral e características limnológicas do ambiente, bem como sobre a classificação do estado trófico do sistema. Nenhum deles, entretanto, faz menção à ocorrência de C. raciborskii, pois se basearam em amostras coletadas até o ano de 1996 e apenas a partir de 1997 (Gentil 2000) C. raciborskii começou a ser encontrada no Lago das Garças, até então dominado por espécies de Microcystis.

Ainda que a herbivoria seja um importante fator regulador de populações fitoplanctônicas em geral, as cianobactérias, por seu tamanho relativamente grande, normalmente não são afetadas por herbívoros (Fulton \& Paerl 1987, Pinto-Coelho et al. 1999) e, portanto, este processo não foi considerado no presente trabalho.

Assim, este estudo teve como objetivo reconhecer os padrões temporais de C. raciborskii, buscando compreender os principais fatores que propiciam sua ocorrência e dominância no lago das Garças.

\section{Material e métodos}

O Lago das Garças foi formado pelo represamento de seis riachos e localiza-se no Parque Estadual das Fontes do Ipiranga (PEFI), zona sul do Município de São Paulo ( $23^{\circ} 39^{\prime} \mathrm{S}$ e $\left.46^{\circ} 37^{\prime} \mathrm{W}\right)$. Encontra-se em altitude média de 786 m e ocupa área de 549 ha. O PEFI é uma das maiores áreas verdes de preservação ambiental em perímetro urbano da cidade de São Paulo, constituindo um dos últimos remanescentes de Mata Atlântica em meio à região densamente urbanizada (Bicudo C. et al. 1999). O clima da região, segundo a classificação de Köppen, é do tipo Cwb, mesotérmico de inverno seco, com temperatura e precipitação médias anuais de $19{ }^{\circ} \mathrm{C}$ e $1.400 \mathrm{~mm}$, respectivamente (Sant'Anna et al. 1989). O Lago das Garças é um ambiente raso, polimítico e eutrófico (Moura 1996, Ramirez 1996, Carmo 2000). Durante o período de estudo, apresentou extensos bancos da macrófita flutuante - Eichhornia crassipes (Mart.) Solms. As características morfométricas do Lago das 
Garças encontram-se descritas por Carmo (2000).

Variações semanais das densidades populacionais do fitoplâncton e das variáveis ambientais foram analisadas a partir de amostragens integradas da coluna d'água realizadas durante o período de setembro/97 a setembro/98. As amostras integradas foram obtidas com tubo de PVC de $2 \mathrm{~m}$ de comprimento por $0,05 \mathrm{~m}$ de diâmetro, na região mais profunda do lago.

Na tabela 1 são apresentadas as variáveis climatológicas, físicas, químicas e biológicas estudadas e a metodologia utilizada. As amostras, para análise dos nutrientes dissolvidos e clorofila $a$, foram filtradas em filtro Whatman $\mathrm{GF} / \mathrm{F}$, previamente calcinados em forno mufla a $450{ }^{\circ} \mathrm{C}$ durante quatro horas para remoção de qualquer composto orgânico eventualmente presente. O limite de detecção dos métodos é de $8,0 \mu \mathrm{g} . \mathrm{L}^{-1}$ para nitrato e de 5,0 $\mu \mathrm{g} . \mathrm{L}^{-1}$ para o amônio e fósforo total.

Considerou-se como zona de mistura $\left(\mathrm{Z}_{\text {mix }}\right)$ a camada de água que se estendeu desde a superfície até aquela em que o gradiente de densidade ultrapassou $0,02 \mathrm{~kg} \cdot \mathrm{m}^{-4}$ ou seja, a profundidade sujeita à mistura turbulenta (Reynolds 1984).

Os cálculos para determinar a concentração de clorofila $a$ seguiram as equações propostas por Lorenzen, apresentadas em Wetzel \& Likens (1991).
Para as análises quantitativas do fitoplâncton, as amostras foram fixadas com lugol acético, quantificadas em cubetas de $2 \mathrm{~mL}$, sendo o tempo de sedimentação de três horas para cada centímetro de altura da câmara (Lund et al. 1958). A contagem dos indivíduos foi realizada em transectos horizontais e verticais e o limite de campos foi determinado de acordo com dois critérios: 1) curva de estabilização do número de espécies, obtida a partir de novas espécies adicionadas com o número de campos contados, e 2) espécies mais abundantes, baseada na contagem de até 100 indivíduos da espécie mais comum.

As unidades amostrais utilizadas nas análises multivariadas (PCA e CCA) foram agrupadas de acordo com as quatro estações do ano, na seguinte sequência: inverno $=10$ e 17 de setembro/97, primavera $=10$ setembro a 17 dezembro/97, verão $=23$ dezembro/97 a 18 março/98, outono $=25$ março a 17 junho/98 e inverno $=24$ junho a 16 setembro/98.

Na tentativa de avaliar a possível influência da presença da macrófita aquática Eichhornia crassipes (Mart.) Solms sobre as variáveis estudadas, adotou-se o seguinte nível de acordo com a cobertura de macrófita: ausência $=0$; presença próxima à estação de amostragem $=1$; e presença na estação de amostragem $=2$.

Tabela 1. Variáveis estudadas e metodologia de análise.

Table 1. Variables studied and analysis methods.

\begin{tabular}{|c|c|c|}
\hline Variáveis & Unidade & Metodologia \\
\hline Precipitação & $\mathrm{mm}$ & $\begin{array}{l}\text { Estação Meteorológica do Observatório do Instituto } \\
\text { Astronômico e Geofísico da USP, localizada no PEFI }\end{array}$ \\
\hline Temperatura do ar & ${ }^{\circ} \mathrm{C}$ & \\
\hline Velocidade do vento & $\mathrm{m} \cdot \mathrm{s}^{-1}$ & \\
\hline Perfil térmico & ${ }^{\circ} \mathrm{C}$ & Termistor \\
\hline Zona de Mistura & $\mathrm{m}$ & Reynolds (1984) \\
\hline Transparência & $\mathrm{m}$ & Disco de Secchi \\
\hline Zona eufótica & $\mathrm{m}$ & Cole (1994) \\
\hline Turbidez & NTU & Turbidímetro \\
\hline Condutividade & $\mu \mathrm{S} . \mathrm{cm}^{-1}$ & Condutivímetro \\
\hline Oxigênio dissolvido $\left(\mathrm{O}_{2}\right)$ & $\mathrm{mg} \cdot \mathrm{L}^{-1}$ & Golterman et al. (1978) \\
\hline $\mathrm{pH}$ & $\mathrm{pH}$ & pHmetro \\
\hline $\mathrm{CO}_{2}$ Total & $\mu \mathrm{g} . \mathrm{L}^{-1}$ & Cole (1979), Wetzel (1981) \\
\hline $\mathrm{CO}_{2}$ Livre & $\mu \mathrm{g} \cdot \mathrm{L}^{-1}$ & Cole (1979), Wetzel (1981) \\
\hline Amônio $\left(\mathrm{N}-\mathrm{NH}_{4}\right)$ & $\mu \mathrm{g} . \mathrm{L}^{-1}$ & Solorzano (1969) \\
\hline Fósforo Total (PT) & $\mu \mathrm{g} . \mathrm{L}^{-1}$ & Valderrama (1981) \\
\hline Clorofila $a$ & $\mu \mathrm{g} . \mathrm{L}^{-1}$ & Sartory \& Grobellar (1984) \\
\hline Densidade & org. $\mathrm{mL}^{-1}$ & Utermöhl (1958) \\
\hline Diversidade (H') & bits.ind ${ }^{-1}$ & Shannon \& Weaver (1963) \\
\hline Dominância (DS) & - & Simpson (1949) \\
\hline Uniformidade (U’) & . & Lloyd \& Gherlardi (1964) \\
\hline Riqueza & to de táxons por amostra & \\
\hline
\end{tabular}


A ordenação dos dados abióticos foi feita através da Análise de Componentes Principais (PCA) (matriz de covariância). A partir do resultado da PCA foram selecionadas as variáveis ambientais $(\mathrm{r}>0,5)$ utilizadas na análise conjunta dos dados abióticos e bióticos, através da análise de correspondência canônica (CCA). O teste de Monte Carlo (99 permutações; $p<0,05$ ) foi usado nesta análise para informar a probabilidade dos autovalores dos eixos terem ou não sido distribuídos ao acaso. Ambas as análises foram realizadas através do programa PC-ORD versão 3.11 para Windows. Os dados foram padronizados pela amplitude de variação ("ranging") através do Programa Fitopac (Shepherd 1996).

\section{Resultados}

Variáveis abióticas - A temperatura do ar oscilou entre o máximo de $27,7^{\circ} \mathrm{C}$ no início do verão (30 Dez/97) e o mínimo de $13{ }^{\circ} \mathrm{C}$ no final do outono (3 Jun/98). A coluna d'água apresentou-se estratificada entre novembro/97 a março/98; em abril/98 a coluna d'água caracterizou-se como um período intermediário, onde as estratificações foram de menor intensidade; entre maio e julho/98 apresentou estratificações e desestratificações. A variação semanal dos perfis de temperatura da coluna d'água está apresentada no diagrama de profundidade e tempo através de isolinhas (figura 1). Na semana na qual o valor máximo de transparência foi registrado, a zona eufótica alcançou o fundo do lago (4,0 m) (figura 2).
De acordo com as figuras 1 e 2, a coluna d'água esteve em geral estratificada durante os meses de primavera e verão com episódios pontuais de mescla total nos meses de setembro, fevereiro e março. Já no outono e inverno o lago esteve totalmente mesclado.

A análise de componentes principais PCA (figura 3) agrupou as semanas de outono e inverno (lado positivo do eixo 1 - figura 3 ) associadas positivamente aos maiores valores de transparência da água, profundidade da zona de mistura e presença da macrófita aquática Eichhornia crassipes e associou-se negativamente com a temperatura da água, turbidez e fósforo total (PT) (tabela 4). Com relação ao eixo 2, a distribuição das unidades amostrais no lado positivo reuniu semanas dos meses de primavera (quando ocorreram floração de $C$. raciborskii) e valores mais elevados de $\mathrm{pH}$ e $\mathrm{O}_{2}$. Já as unidades amostrais localizadas no lado negativo do eixo, também representadas por semanas dos meses de primavera e verão, apresentaram altas concentrações de $\mathrm{N}-\mathrm{NH}_{4} \mathrm{e}$ $\mathrm{CO}_{2}$ Livre (tabela 4).

Variáveis biológicas - Os tricomas de C. raciborskii caracterizaram-se por apresentar desde 8-10 até 85 células. Menos de $9 \%$ dos tricomas encontrados foram portadores de heterocito. Durante o período de estudo, os tricomas encontrados não apresentaram formação de acinetos.

Foram identificados 193 táxons distribuídos em 10 classes. Chlorophyceae foi a classe com maior riqueza

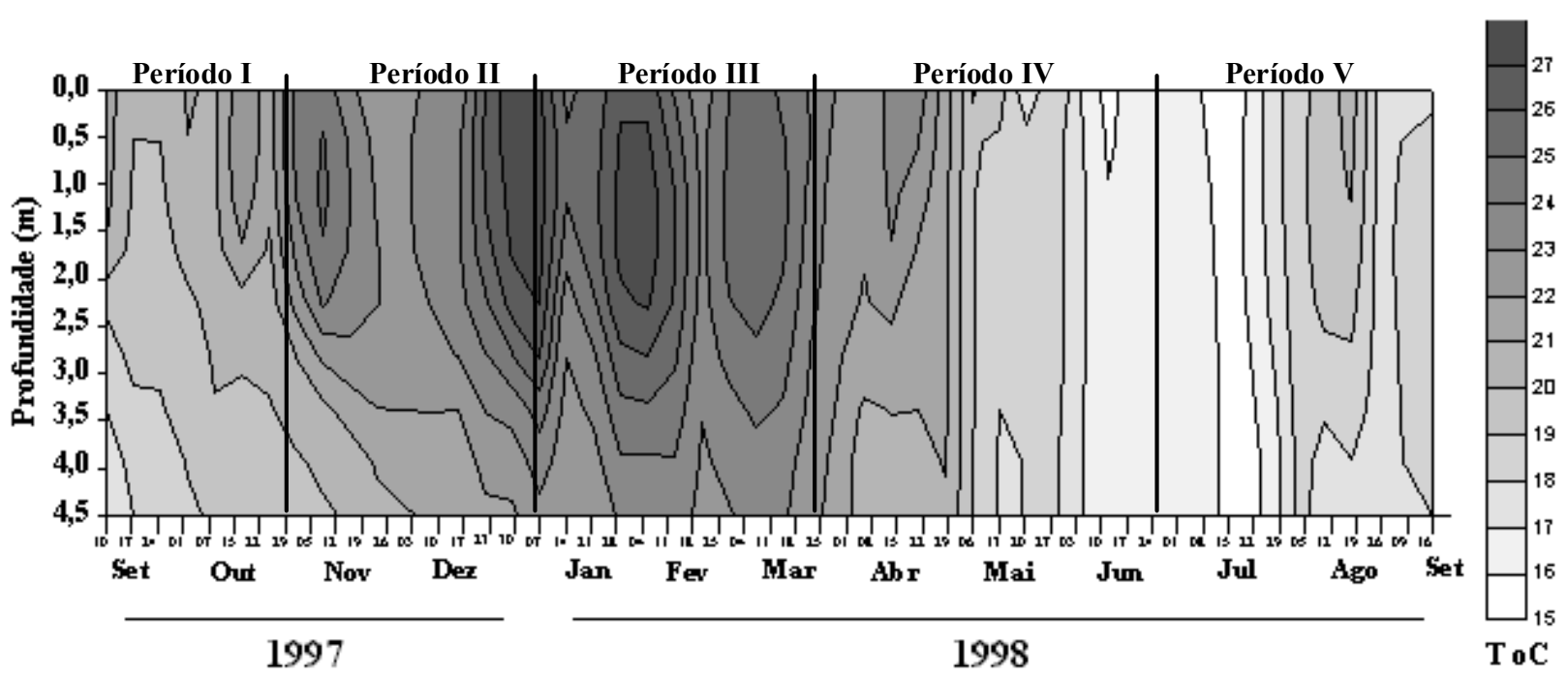

\section{Semanas}

Figura 1. Diagrama de profundidade $(\mathrm{m})$ e tempo das isolinhas de temperatura da água $\left({ }^{\circ} \mathrm{C}\right)$ no Lago das Garças, no período estudado (Período I - primavera; Período II - primavera; Período III - verão; Período IV - outono; Período V - inverno).

Figure 1. Deepness diagram $(\mathrm{m})$ and timecourse of water temperature $\left({ }^{\circ} \mathrm{C}\right)$ in the "Lago das Garças" during the period of study (Period I - Spring; Period II - Spring; Period III - Summer; Period IV - Autumn; Period V - Winter). 


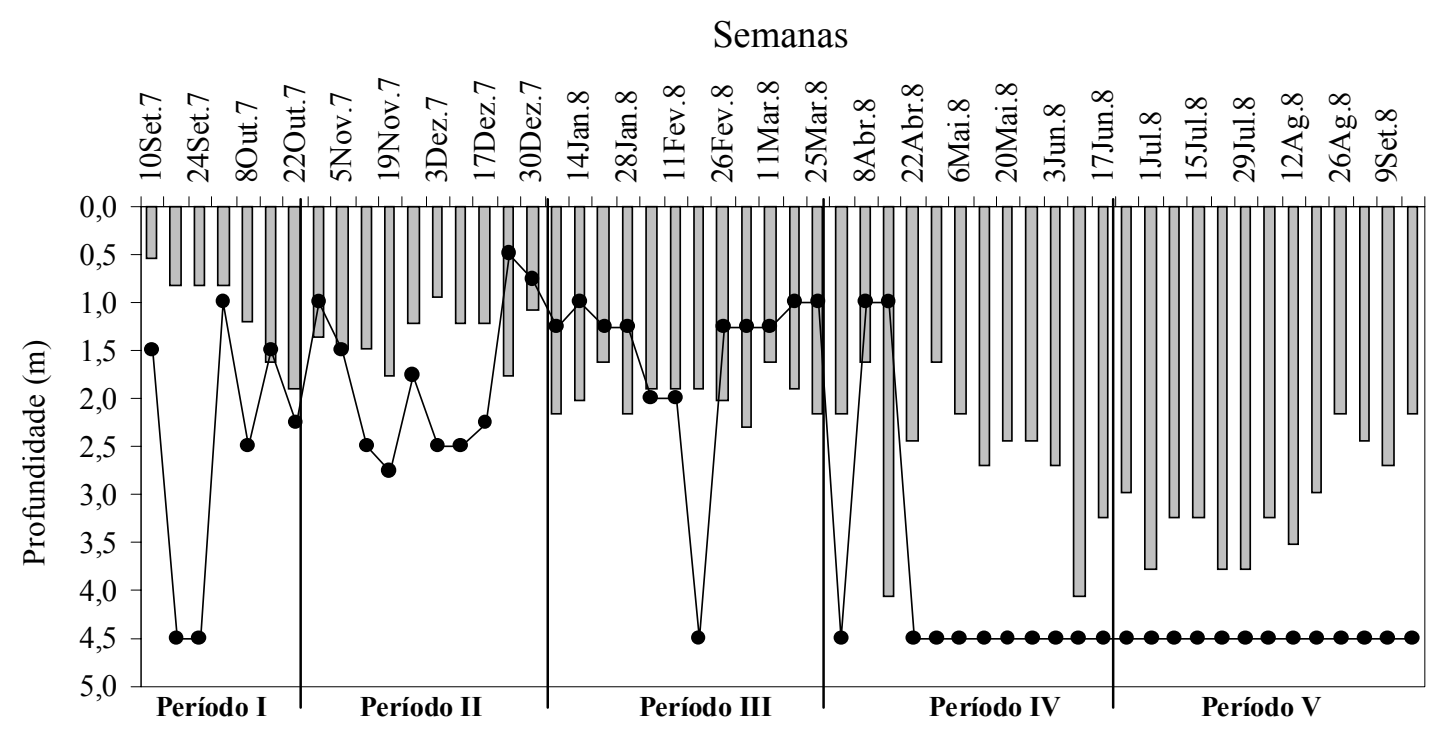

Figura 2. Variação semanal da profundidade de zona eufótica $(Z e u f=\square)(\mathrm{m})$ e da profundidade de zona de mistura $($ Zmix $=$ $-\bullet$ ) (m) para a estação de amostragem no período de estudo(Período I - primavera; Período II - primavera; Período III verão; Período IV - outono; Período V - inverno).

Figure 2. Weeky variation of the euphotic zone deepness $(\mathrm{Zeuf}=\square)(\mathrm{m})$ and mixing zone deepness $(\mathrm{Zmix}=-\bullet)(\mathrm{m})$ for the sampling station along the period of study (Period I - Spring; Period II - Spring; Period III - Summer; Period IV - Autumn; Period V - Winter).

de táxons (90), seguida por Cyanobacteria (28) e Euglenophyceae (22).

A variação semanal da biomassa da comunidade, medida através da concentração de clorofila $a$, está apresentada na figura 4. A densidade máxima da comunidade fitoplanctônica foi de 90.800 org. $\mathrm{mL}^{-1}$ e a densidade máxima de $C$. raciborskii foi de 21.450 org. $\mathrm{mL}^{-1}$ (figura 5), o que equivale a 210.450 células $/ \mathrm{mL}$ (considerando que o número médio de células por tricoma para o lago das Garças foi 10). As variações semanais da densidade de $C$. raciborskii e da densidade da comunidade, exceto C. raciborskii, estão apresentadas na figura 5 .

Ao longo do período de estudo, a espécie contribuiu, em média, com apenas $3 \%$ da densidade total anual do fitoplâncton. Entretanto, destacou-se durante oito semanas, em meses de primavera $(05 / 11$ a $30 / 12 / 97)$, e foi classificada nas semanas de 10/12 a $17 / 12$ como dominante, chegando a representar mais de $50 \%$ da densidade total da comunidade (figura 5).

Chlorophyceae, Cryptophyceae e Cyanobacteria foram as classes que mais contribuíram para o fitoplâncton total ao longo do estudo e juntas representaram, em média, $90 \%$ da densidade total. As variações semanais das densidades destas classes estão apresentadas na figura 6 . As menores densidades foram das classes Prasinophyceae, Dinophyceae e Xanthophyceae.

Além de Cylindrospermopsis raciborskii, somente duas outras espécies foram classificadas como dominantes ao longo de todo período de estudo: Choricystis minor (Skuja) Fott var. gallica (Bourrelly) Komárek (Chlorophyceae) e Cryptomonas curvata Ehrenberg emend. Penard (Cryptophyceae). Essas três espécies somaram, em média, $30 \%$ da densidade total anual da comunidade. Cabe ressaltar que as duas espécies constituem novas ocorrências para o ambiente.

Assim, com base nas variações da composição e densidade fitoplanctônicas, foram identificados cinco períodos descritos a seguir:

Período I - 10/09 a 29/10/97 - primavera: elevada densidade, com dominância de cianobactérias, baixa transparência e coluna d'água geralmente estratificada. Período II - 5/11 a 30/12/97 - primavera: densidades intermediárias, dominância de $C$. raciborskii, baixa transparência e coluna d'água geralmente estratificada. Período III - 7/01 a 25/03/98 - verão: densidades intermediárias, co-dominância de cianobactérias e criptofíceas, ausência de $C$. raciborskii, transparências intermediárias e coluna d'água estratificada.

Período IV - 7/04 a 24/06/98 - outono: densidades intermediárias, co-dominância de clorofíceas e 


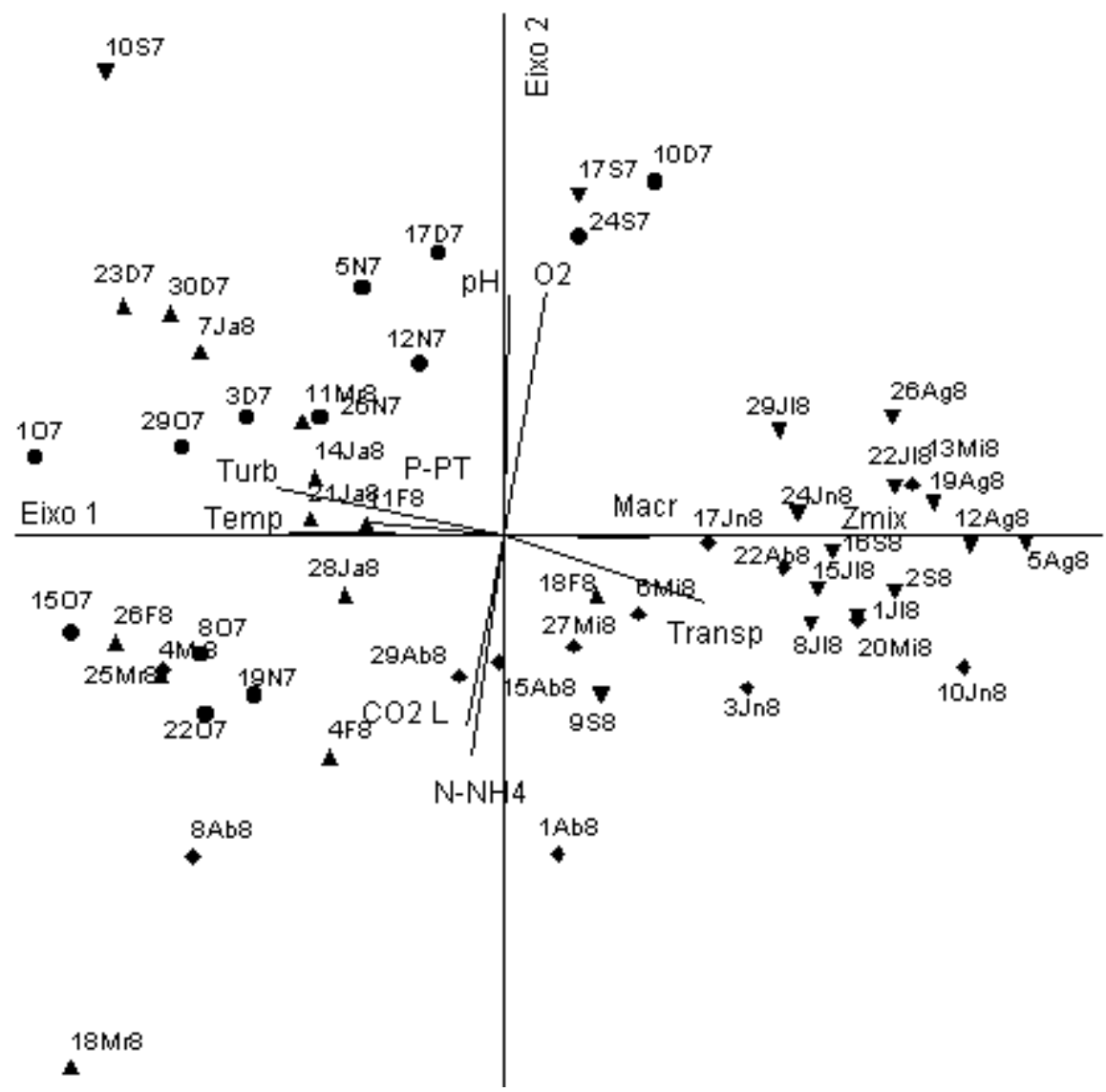

Figura 3. Ordenação pela PCA, das unidades amostrais (semanas) e das variáveis físicas e químicas analisadas. As unidades amostrais foram identificadas de acordo com as épocas do ano: Primavera $=\bullet(24 \mathrm{~S} 7$ a $17 \mathrm{D} 7)$, Verão $=\boldsymbol{\bullet}(23 \mathrm{D} 7$ a $18 \mathrm{Mr} 8)$, Outono $=\bullet(25 \mathrm{Mr} 8$ a $17 \mathrm{Jn} 8)$ e Inverno $=\boldsymbol{\nabla}(10 \mathrm{~S} 7$ e 17S7; 24Jn8 a 16S8). Os números localizados na frente das unidades amostrais, correspondem ao dia do mês e os números 7 e 8 no final das unidades correspodem respectivamente aos anos de 1997 e 1998. Os meses estão abreviados como: $\mathrm{Ja}=\mathrm{Janeiro,} \mathrm{F}=$ fevereiro, $\mathrm{Mr}=$ março, $\mathrm{Ab}=$ abril, $\mathrm{Mi}=$ maio, $\mathrm{Jn}=$ junho, $\mathrm{Jl}$ = julho, $\mathrm{Ag}=$ agosto, $\mathrm{S}=$ setembro, $\mathrm{O}=$ outubro, $\mathrm{N}=$ novembro, $\mathrm{D}=$ dezembro. As abreviações das variáveis ambientais estão apresentadas na tabela 4.

Figure 3. PCA ordination of units (weeks) and of analysed physical and chemical variables. The units were identified based on seasons: Spring $=\bullet$ (24S7 to 17D7), Summer $=\boldsymbol{\bullet}(23 \mathrm{D} 7$ to $18 \mathrm{Mr})$, Autumn $=\bullet(25 \mathrm{Mr} 8$ to 17Jn8) and Winter $=\boldsymbol{\nabla}(10 \mathrm{~S} 7$ and 17S7; 24Jn8 to 16S8). The numbers in front of units correspond to the day of the month and the numbers 7 and 8 at the end of the units correspond to the years 1997 and 1998. Months are abbreviated as $\mathrm{Ja}=\mathrm{January} ; \mathrm{F}=\mathrm{February} ; \mathrm{Mr}=\mathrm{March} ; \mathrm{Ab}=$ April; $\mathrm{Mi}=$ May; $\mathrm{Jn}=\mathrm{June} ; \mathrm{Jl}=\mathrm{July} ; \mathrm{Ag}=$ August; $\mathrm{S}=$ September; $\mathrm{O}=$ October; $\mathrm{N}=$ November; $\mathrm{D}=$ December. Abbreviations of the environmental variables are presented in the table 4.

cianobactérias, ausência de C. raciborskii, transparências intermediárias e crescente, com desestratificação térmica da coluna d'água.

Período V - 1/07 a 16/09/98 - inverno: densidades elevadas de células muito pequenas, dominância de clorofíceas, alta transparência e desestratificação térmica da coluna d'água.

O resultado da análise de correspondência canônica está apresentado na figura 7 e nas tabelas 3 e 5 . O teste de Monte Carlo foi significativo $(\mathrm{p}<0,05)$, o que indica que os acontecimentos não foram ao acaso, representando as relações existentes entre as variáveis ambientais e as informações biológicas. Assim, os coeficientes canônicos para o eixo 1 (tabela 5) mostraram que a transparência da água foi a variável de maior peso na ordenação. As correlações "intra-set" (tabela 5) indicaram, por sua vez, que além da transparência, a profundidade de zona de mistura também contribuiu na relação espécie-ambiente.

A CCA indicou que as unidades amostrais nos quais 
foram registradas florações de $C$. raciborskii (semanas da primavera - lado positivo do eixo 1) estiveram associadas a valores elevados de $\mathrm{pH}$, altas concentrações de clorofila $a$, altas densidades de outras cianobactérias, e baixos valores de transparência da água, profundidade de zona de mistura, concentrações de gás carbônico livre e amônio (figura 7, tabelas 3,5).

Os atributos da comunidade fitoplanctônica indicaram que, mesmo durante dominância de C. raciborskii (período II - primavera), a riqueza, a diversidade e a uniformidade das espécies em média se mantiveram constantes. Os valores máximos de riqueza e diversidade de espécies foram registrados no outono. Os menores valores de riqueza foram registrados na primavera e baixa diversidade foi registrada no inverno (tabela 6). A queda na riqueza (primavera) coincidiu com as quedas na concentração de clorofila $a$ e na densidade total da comunidade (figuras $4,5,6$ ).

$\mathrm{O}$ índice de dominância apresentou variações acentuadas ao longo do período de estudo, indicado pelo maior valor de coeficiente de variação (tabela 6). Valores elevados do índice de dominância $(0,63)$ foram registrados durante o período de grande desenvolvimento da Chlorophyceae Choricystis minor (Skuja) Fott var. gallica (Bourrelly) Komárek (período V - inverno).

\section{Discussão}

As análises multivariadas (PCA e CCA) permitiram a interpretação conjunta dos dados abióticos e bióticos, os quais mostraram que durante a floração de C. raciborskii (primavera) foram registradas, em média, altas temperatura da água, turbidez, $\mathrm{pH}$ e oxigênio dissolvido. Além disso, observaram-se alta disponibilidade de nitrogênio na forma do íon amônio e baixos valores de transparência da água, profundidade de mistura, além da ausência da macrófita $E$. crassipes na estação de coleta. Assim sendo, consideramos que estes foram os fatores fundamentais para $o$ desenvolvimento dessa floração no Lago das Garças.

Padisák (1997) mencionou que o sucesso ecológico de $C$. raciborskii está diretamente relacionado aos seguintes fatores: capacidade de migração na coluna d'água, tolerância à baixa luminosidade, habilidade em utilizar fontes internas de fósforo, alta afinidade com fósforo e amônio, capacidade de fixar nitrogênio atmosférico, resistência à herbivoria pelo zooplâncton, alta capacidade de dispersão (acinetos resistentes, dispersão por cursos de rios, aves, etc.) e sobrevivência em condições levemente salinas. Desses fatores, dois foram observados no presente trabalho: tolerância à

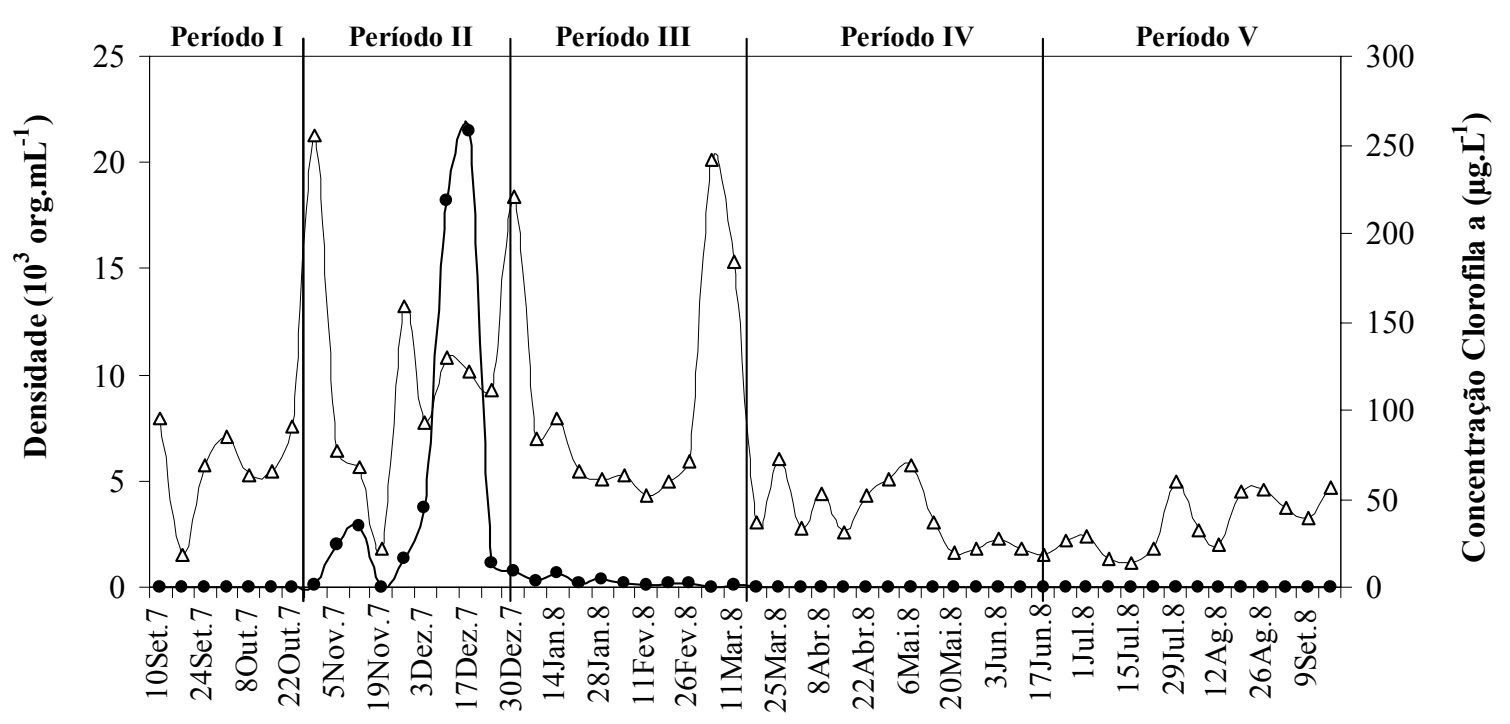

Semanas

Figura 4. Variação semanal da densidade (org.mL $\left.\mathrm{L}^{-1}\right)$ de $C$. raciborskii $(-\bullet-)$ e da concentração de clorofila $a($ Cloro $a=-\triangle-)\left(\mu g . \mathrm{L}^{-1}\right)$, durante o período de estudo (Período I - primavera; Período II - primavera; Período III - verão; Período IV - outono; Período V inverno).

Figure 4. Weekly variation of the density (org. $\left.\mathrm{mL}^{-1}\right)$ of $C$. raciborskii $(-\bullet-)$ and of the $a$-chlorophyll concentration $($ Cloro $a=$ $-\triangle-)\left(\mu \mathrm{g} . \mathrm{L}^{-1}\right)$ during the period of study (Period I - Spring; Period II - Spring; Period III - Summer; Period IV - Autumm; Period $\mathrm{V}-$ Winter). 


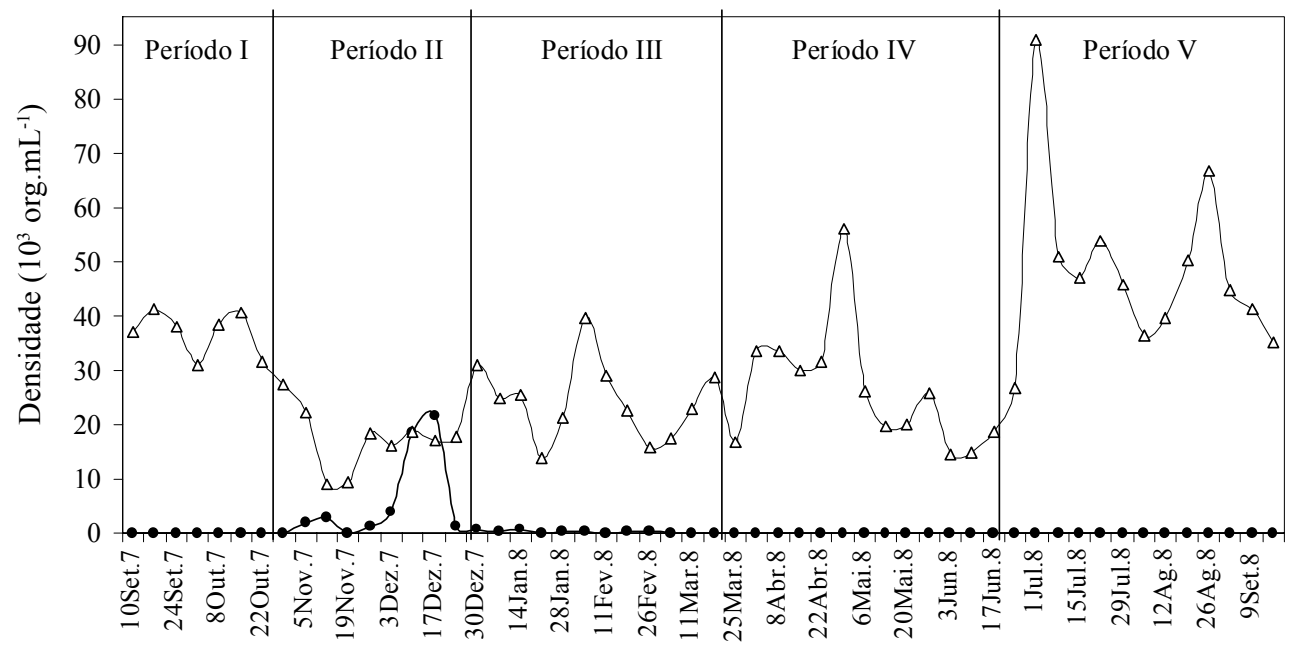

Semanas

Figura 5. Variação semanal da densidade (org. $\left.\mathrm{mL}^{-1}\right)$ de C. raciborskii $(-\bullet-)$ e da densidade total $\left(\mathrm{Dt}=-\triangle_{-}\right)$da comunidade (sem a referida espécie) (org. $\mathrm{mL}^{-1}$ ), durante o período de estudo (Período I - primavera; Período II - primavera; Período III verão; Período IV - outono; Período V - inverno).

Figure 5. Weekly variation in density (org. $\left.\mathrm{mL}^{-1}\right)$ of C. raciborskii $(-\bullet-)$ and total density $(\mathrm{Dt}=-\triangle-$ ) of the community (without this species) (org. $\mathrm{mL}^{-1}$ ), during the study period. (Period I - Spring; Period II - Spring; Period III - Summer; Period IV Autumn; Period V - Winter).

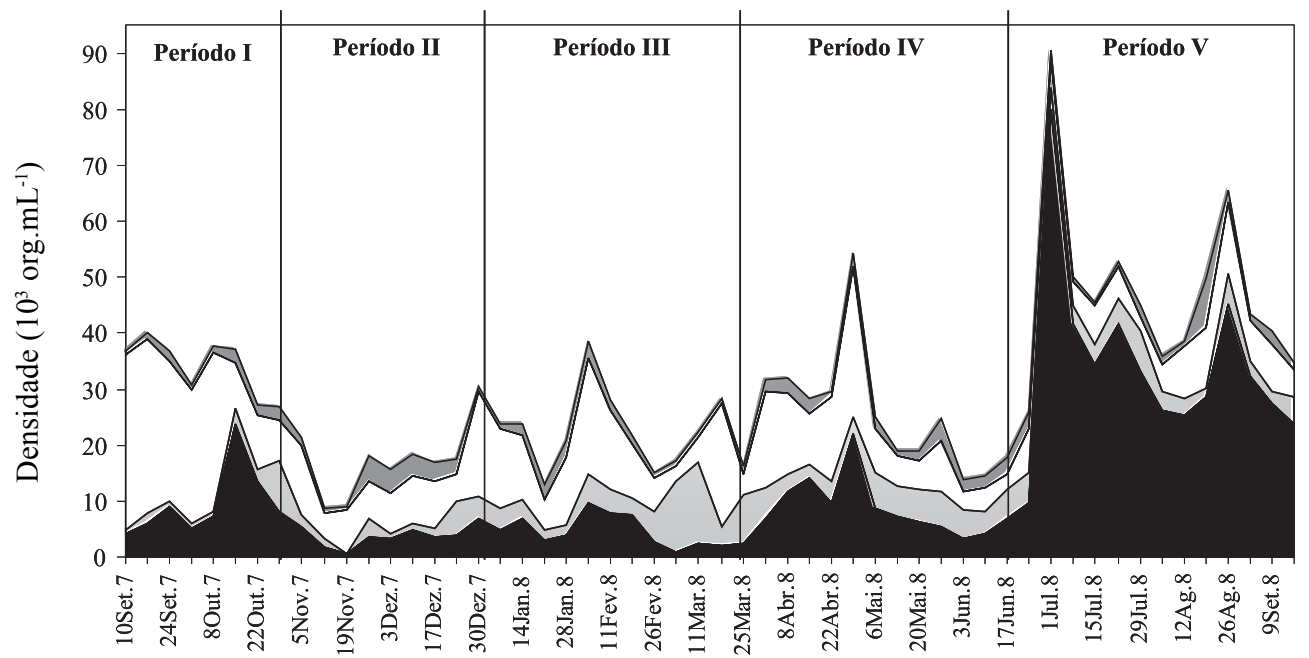

Semanas

Figura 6. Variação semanal da densidade (org. $\mathrm{mL}^{-1}$ ) das classes fitoplanctônicas representativas da comunidade durante o período de estudo (Chloro $\boldsymbol{\square}=$ Chlorophyceae, Crypto $\square=$ Cryptophyceae, Cyano $\square=$ Cyanophyceae e Outros $\square=$ Bacillariophyceae, Chrysophyceae, Dinophyceae, Prasinophyceae, Xanthophyceae e Zygnemaphyceae. (Período I- primavera; Período II - primavera; Período III - verão; Período IV - outono; Período V - inverno).

Figure 6. Weekly variation of the density (org. $\mathrm{mL}^{-1}$ ) of the main phytoplanktonic classes, during the study period (Chloro $\mathbf{\square}=$ Chlorophyceae, Crypto $\square=$ Cryptophyceae, Cyano $\square=$ Cyanophyceae and others $\square=$ Bacillariophyceae, Chrysophyceae, Dinophyceae, Prasinophyceae, Xanthophyceae and Zygnemaphyceae. (Period I - Spring; Period II - Spring; Period III Summer; Period IV - Autumn; Period V - Winter). 


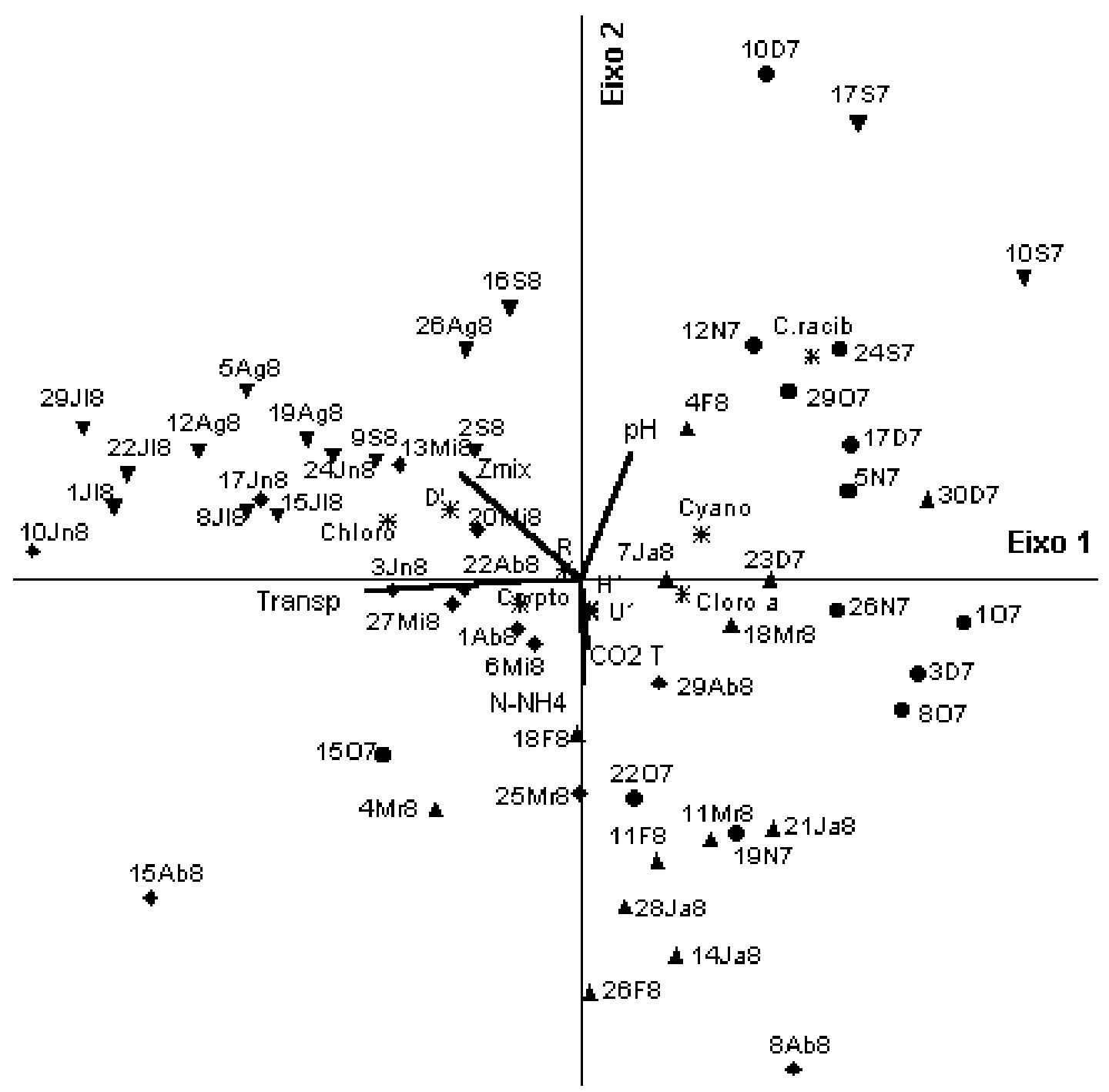

Figura 7. Ordenação pela CCA das unidades amostrais no Lago das Garças, baseada em seis variáveis ambientais e nove variáveis biológicas. Variáveis ambientais: transparência (Transp), $\mathrm{pH}(\mathrm{pH})$, zona de mistura (Zmix), amônio (N-NH4), gás carbonico total (CO2 T). Variáveis biológicas: C. racisborskii (C.racib), clorofila a (Cloro a), Chlorophyceae (Chloro), Cryptophyceae (Crypto), Cyanophyceae (Cyano), riqueza (R), diversidade (H'), uniformidade (U') e dominância (D'). Unidades amostrais: As unidades amostrais foram identificadas de acordo com as épocas do ano: Primavera $=\bullet(24 \mathrm{~S} 7$ a $17 \mathrm{D} 7)$, Verão $=$ - $(23 \mathrm{D} 7$ a $18 \mathrm{Mr} 8)$, Outono $=\bullet(25 \mathrm{Mr} 8 \mathrm{a} 17 \mathrm{Jn} 8)$ e Inverno $=\boldsymbol{\nabla}(10 \mathrm{~S} 7$ e $17 \mathrm{~S} 7 ; 24 \mathrm{Jn} 8$ a $16 \mathrm{~S} 8)$. Os números localizados na frente das unidades amostrais, correspondem ao dia do mês e os números 7 e 8 no final das unidades correspodem respectivamente aos anos de 1997 e 1998. Os meses estão abreviados como: $\mathrm{Ja}=$ Janeiro, $\mathrm{F}=$ fevereiro, $\mathrm{Mr}=$ março, $\mathrm{Ab}=$ abril, $\mathrm{Mi}=$ maio, $\mathrm{Jn}=$ junho, $\mathrm{Jl}=$ julho, $\mathrm{Ag}=$ agosto, $\mathrm{S}=$ setembro, $\mathrm{O}=$ outubro, $\mathrm{N}=$ novembro, $\mathrm{D}=$ dezembro.

Figure 7. PCA ordination of the samples from "Lago das Garças" based on six environmental variables and nine biological variables. Environmental variables: transparency (Transp), $\mathrm{pH}(\mathrm{pH})$, mixing zone (Zmix), ammonium (N-NH4), total carbon dioxide (CO2 T). Biological variables: C. racisborskii (C.racib), $a$-clorophyll (Cloro $a$ ), Chlorophyceae (Chloro), Cryptophyceae (Crypto), Cyanophyceae (Cyano), richness (R), diversity (H'), uniformity (U') and dominance (D'). Sample units: the sample units were identified according to the seasons: Spring $=\bullet(24 \mathrm{~S} 7$ to $17 \mathrm{D} 7)$, Summer $=\boldsymbol{\bullet}(23 \mathrm{D} 7$ to $18 \mathrm{Mr} 8)$, Autumn $=\boldsymbol{\bullet}(25 \mathrm{Mr} 8$ to $17 \mathrm{Jn} 8)$ and Winter $=\boldsymbol{\nabla}(10 \mathrm{~S} 7$ and $17 \mathrm{~S} 7 ; 24 \mathrm{Jn} 8$ to $16 \mathrm{~S} 8)$. The numbers in front of sample units correspond to the day of the month and the numbers 7 and 8 at the end of the units correspond to the years 1997 and 1998, respectively. Months are abbreviated as $\mathrm{Ja}=$ January; $\mathrm{F}=$ February; $\mathrm{Mr}=$ March; $\mathrm{Ab}=$ April; Mi = May; $\mathrm{Jn}=\mathrm{June} ; \mathrm{Jl}=\mathrm{July} ; \mathrm{Ag}=$ August; $\mathrm{S}=$ September; $\mathrm{O}=$ October; $\mathrm{N}=$ November; $\mathrm{D}=$ December. 
baixa luminosidade e alta afinidade com amônio.

Ao acompanharem a variação sazonal de C. raciborskii no Rio Pequeno, braço da Represa Billings, SP, Souza et al. (1998) concluíram que o desenvolvimento da espécie foi favorecido por valores elevados de temperatura da água, do $\mathrm{pH}$ e da concentração de oxigênio dissolvido. Condições semelhantes também foram observadas no Lago das Garças durante o desenvolvimento da floração de C. raciborskii.

Branco \& Cavalcanti (1999) comentaram que entre as hipóteses para explicar a dominância de $C$. raciborskii no Lago Paranoá, levantadas por Pinto-Coelho (1983), Alves et al. (1988) e Starling (1989), estão incluídos os seguintes fatores: migração na coluna d'água, fixação de nitrogênio atmosférico e resistência à herbivoria do zooplâncton. Com relação à migração na coluna d'água, sabe-se que os aerótopos presentes nas células de C. raciborskii possibilitam a sua movimentação na zona eufótica, dependendo das condições de luminosidade e necessidades metabólicas das células (Padisák 1997). No caso do lago das Garças, a migração de C. raciborskii na coluna d'água não pode ser descartada uma vez que sua dominância ocorreu durante o período de estratificação térmica, associada à baixa profundidade de mistura porém dentro da zona eufótica.

Dos fatores discutidos em literatura para justificar o desenvolvimento de $C$. raciborskii, alguns não foram observados no presente trabalho. Entre eles estão a herbivoria e a fixação de nitrogênio atmosférico. Quanto à herbivoria pode-se dizer que as cianofíceas em geral costumam ser evitadas em virtude de suas elevadas dimensões, além de que os organismos zooplanctônicos têm preferência por grupos mais palatáveis, principalmente clorofíceas e criptofíceas (Pinto-Coelho 1983, Branco \& Senna 1994, Padisák 1997).

No caso do Lago das Garças, a fixação de nitrogênio atmosférico pode ser descartada como estratégia para o intenso desenvolvimento de C. raciborskii, pois somente um pequeno número de tricomas $(9 \%)$ formou heterocito. Isto se deve ao fato do nitrogênio nunca ser limitante no lago, sobretudo o íon amônio (tabela 2) que é a forma preferencial de absorção do nitrogênio (Saker \& Neilan 2001). Desta forma, não há necessidade de gastos energéticos para formação de heterocitos.

Baixo percentual de tricomas portadores de heterocitos foram também documentados em ambientes com altas concentrações de nitrogênio, em diferentes regiões do Brasil, como para o Lago Paranoá (DF) (Branco \& Senna 1994), para açudes do nordeste do
Brasil (Huszar et al. 2000) e para um reservatório raso e eutrófico do semi-árido de Pernambuco (Bouvy et al. 1999).

No outono e inverno, períodos IV e V, respectivamente, a ausência de $C$. raciborskii no Lago das Garças (figura 6), está relacionada a condições ambientais como: 1) baixas temperaturas do ar e da água, 2) longos períodos de desestratificação térmica da coluna d'água e aumento na profundidade da zona de mistura; tais condições ambientais também foram discutidas por Komárková et al. (1999) e Padisák (1997). Além disso, o desenvolvimento acelerado dos bancos de macrófitas acarretou altos valores de transparência (os maiores já registrados para o lago estudado), fato que normalmente não favorece o desenvolvimento de cianobactérias. A influência das macrófitas sobre as condições físicas e químicas da água do lago das Garças foi anteriormente registrada por Carmo (2000) que concluiu que os valores de concentração de NT e PT, em 1997 e 1998, foram menores quando comparados aos dados de anos anteriores apresentados por Ramirez (1996) e Nogueira (1997). Segundo Carmo (2000), o principal responsável pelas alterações físicas e químicas da água foi a grande expansão dos bancos de Eichhornia crassispes, uma vez que não se constatou tendência de redução das cargas de nutrientes que entravam no sistema. E. crassipes é uma boa indicadora de ambientes eutróficos e sua importância reside em seu papel no estoque e ciclagem dos nutrientes, na sua interferência na redução da penetração de luz na água e na competição com a comunidade fitoplanctônica por nutrientes (Beyruth 1992, 1994), o que foi constatado para o Lago das Garças.

Com relação à sucessão das classes fitoplanctônicas, Cyanobacteria foi dominante em algumas semanas durante os períodos I, II e III primavera até final do outono/98. Essa dominância não foi contínua mas alternada com Chlorophyceae e Cryptophyceae. Durante todo o período V - inverno/98, Chlorophyceae foi a classe dominante, sendo representada quase que exclusivamente por uma pequena Chlorococcales unicelular Choricystis minor (Skuja) Fott var. gallica (Bourrelly) Komárek.

Ao contrário do esperado, a floração de C. raciborskii não implicou em queda acentuada na diversidade, riqueza, dominância e uniformidade, que praticamente permaneceram constantes. Os táxons que coexistiram com $C$. raciborskii foram espécies de Cryptomonas (Cryptophyceae) e outras Cyanobacteria (Coelosphaerium evidenter-marginatum Azevedo \& 
Tabela 2. Valores semanais das variáveis analisadas: turbidez (Turb), condutividade (Cond), $\mathrm{pH}$, oxigênio dissolvido $\left(\mathrm{O}_{2}\right)$, gás carbônico total $\left(\mathrm{CO}_{2} \mathrm{~T}\right)$ e livre $\left(\mathrm{CO}_{2} \mathrm{~L}\right)$, fósforo total $(\mathrm{PT})$ e amônio $\left(\mathrm{NH}_{4}\right)$, durante o período de estudo $(\mathrm{M}=$ macrófita nas proximidades da estação de coleta, $\mathrm{ME}$ = macrófita na estação de coleta, $\mathrm{A}$ = macrófita ausente na estação de coleta).

Table 2. Values obtained weekly for the following variables: turbidity (Turb), conductivity (Cond), $\mathrm{pH}$, dissolved oxygen $\left(\mathrm{O}_{2}\right)$, total carbon dioxide $\left(\mathrm{CO}_{2} \mathrm{~T}\right)$ free carbon dioxide $\left(\mathrm{CO}_{2} \mathrm{~L}\right)$, total phosphorus $(\mathrm{PT})$ and ammonium $\left(\mathrm{NH}_{4}\right)$, during the period of study $(\mathrm{M}=$ macrophyte from the sampling station surroundings, $\mathrm{ME}=$ macrophyte from the sampling station, $\mathrm{A}=$ macrophyte absent in the sampling station).

\begin{tabular}{|c|c|c|c|c|c|c|c|c|c|c|}
\hline & Semanas & Macrófita & $\begin{array}{l}\text { Turb } \\
\text { (NTU) }\end{array}$ & $\begin{array}{l}\text { Cond } \\
\left(\mu \mathrm{S} . \mathrm{cm}^{-1}\right)\end{array}$ & $\begin{array}{l}\mathrm{pH} \\
(\mathrm{pH})\end{array}$ & $\begin{array}{c}\mathrm{O}_{2} \\
\left(\mu \mathrm{g} \cdot \mathrm{L}^{-1}\right)\end{array}$ & $\begin{array}{c}\mathrm{CO}_{2} \mathrm{~T} \\
\left(\mu \mathrm{g} \cdot \mathrm{L}^{-1}\right)\end{array}$ & $\begin{array}{l}\mathrm{CO}_{2} \mathrm{~L} \\
\left(\mu \mathrm{g} . \mathrm{L}^{-1}\right)\end{array}$ & $\begin{array}{c}\mathrm{PT} \\
\left(\mu \mathrm{g} \cdot \mathrm{L}^{-1}\right)\end{array}$ & $\begin{array}{c}\mathrm{NH}_{4} \\
\left(\mu \mathrm{g} \cdot \mathrm{L}^{-1}\right)\end{array}$ \\
\hline \multirow{8}{*}{ 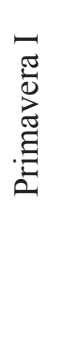 } & 10/9/97 & A & 35 & 103 & 7,6 & 6,4 & 30,9 & 1,6 & 196 & 12 \\
\hline & $17 / 9 / 97$ & M & 28 & 100 & 7,4 & 5,9 & 35,3 & 2,8 & 146 & 57 \\
\hline & 24/9/97 & M & 28 & 96 & 7,1 & 6,7 & 37,4 & 6,1 & 148 & 127 \\
\hline & 1/10/97 & M & 30 & 158 & 7,1 & 0,7 & 41,9 & 6,4 & 182 & 199 \\
\hline & 8/10/97 & M & 20 & 134 & 7,0 & 0,5 & 42,6 & 7,5 & 105 & 684 \\
\hline & $15 / 10 / 97$ & A & 16 & 162 & 7,2 & 0,3 & 41,7 & 5,0 & 508 & 791 \\
\hline & $22 / 10 / 97$ & A & 16 & 171 & 7,1 & 0,2 & 44,7 & 6,7 & 169 & 1.052 \\
\hline & 2910/97 & $\mathrm{M}$ & 17 & 147 & 7,5 & 0,7 & 37,1 & 2,4 & 232 & 146 \\
\hline \multirow{9}{*}{ 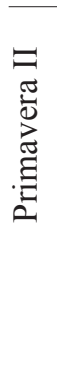 } & $5 / 11 / 97$ & $\mathrm{M}$ & 18 & 127 & 7,2 & 5,8 & 39,3 & 4,7 & 106 & 71 \\
\hline & $12 / 11 / 97$ & M & 17 & 150 & 7,3 & 3,4 & 41,4 & 4,2 & 155 & 95 \\
\hline & $19 / 11 / 97$ & A & 18 & 167 & 6,8 & 0,9 & 52,2 & 13,3 & 73 & 958 \\
\hline & $26 / 11 / 97$ & M & 19 & 154 & 7,2 & 6,3 & 40,7 & 5,2 & 171 & 446 \\
\hline & $3 / 12 / 97$ & A & 19 & 147 & 6,9 & 4,9 & 45,3 & 9,4 & 119 & 474 \\
\hline & $10 / 12 / 97$ & M & 19 & 144 & 7,4 & 7,4 & 38,3 & 2,9 & 134 & 26 \\
\hline & $17 / 12 / 97$ & M & 17 & 144 & 7,1 & 6,0 & 43,3 & 6,8 & 133 & 10 \\
\hline & $23 / 12 / 97$ & A & 19 & 119 & 7,2 & 4,4 & 39,1 & 4,4 & 155 & 98 \\
\hline & $30 / 12 / 97$ & A & 8 & 134 & 7,3 & 3,5 & 36,2 & 3,4 & 169 & 17 \\
\hline \multirow{12}{*}{ 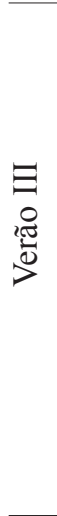 } & $7 / 1 / 98$ & $\mathrm{~A}$ & 15 & 145 & 7,1 & 4,2 & 35,9 & 4,9 & 115 & 17 \\
\hline & $14 / 1 / 98$ & M & 15 & 141 & 6,3 & 6,8 & 67,3 & 34,6 & 117 & 410 \\
\hline & $21 / 1 / 98$ & M & 15 & 130 & 6,1 & 4,2 & 76,5 & 46,8 & 113 & 84 \\
\hline & $28 / 1 / 98$ & $\mathrm{ME}$ & 14 & 125 & 6,4 & 2,6 & 54,8 & 26,2 & 121 & 94 \\
\hline & $4 / 2 / 98$ & M & 14 & 128 & 5,7 & 1,2 & 132,9 & 105,2 & 94 & 47 \\
\hline & $11 / 2 / 98$ & M & 14 & 127 & 6,3 & 3,5 & 58,4 & 31,6 & 121 & 49 \\
\hline & $18 / 2 / 98$ & M & 17 & 147 & 6,9 & 4,4 & 44,5 & 9,6 & 116 & 1.036 \\
\hline & $26 / 2 / 98$ & A & 17 & 116 & 6,2 & 3,0 & 72,9 & 42,0 & 184 & 539 \\
\hline & $4 / 3 / 98$ & M & 15 & 129 & 6,1 & 1,3 & 80,2 & 50,3 & 261 & 62 \\
\hline & $11 / 3 / 98$ & M & 16 & 126 & 6,5 & 4,2 & 50,9 & 22,7 & 181 & 28 \\
\hline & $18 / 3 / 98$ & M & 21 & 153 & 5,8 & 0,4 & 164,2 & 126,2 & 105 & 1.285 \\
\hline & $25 / 3 / 98$ & A & 14 & 137 & 6,0 & 4,8 & 111,3 & 77,7 & 173 & 588 \\
\hline \multirow{13}{*}{$\begin{array}{l}\geq \\
0 \\
0 \\
0 \\
\stackrel{0}{\Xi} \\
0\end{array}$} & $1 / 4 / 98$ & $\mathrm{M}$ & 16 & 143 & 6,1 & 1,6 & 99,1 & 65,1 & 81 & 794 \\
\hline & $8 / 4 / 98$ & $\mathrm{M}$ & 15 & 142 & 6,1 & 2,2 & 97,3 & 62,7 & 106 & 1.134 \\
\hline & $15 / 4 / 98$ & $\mathrm{ME}$ & 13 & 142 & 6,7 & 2,5 & 42,9 & 12,6 & 119 & 307 \\
\hline & $22 / 4 / 98$ & ME & 14 & 134 & 6,8 & 4,2 & 44,1 & 12,0 & 82 & 426 \\
\hline & $29 / 4 / 98$ & A & 15 & 142 & 6,7 & 0,7 & 48,5 & 15,2 & 96 & 651 \\
\hline & $6 / 5 / 98$ & M & 15 & 142 & 6,8 & 3,1 & 44,4 & 12,9 & 82 & 548 \\
\hline & $13 / 5 / 98$ & $\mathrm{ME}$ & 3 & 142 & 6,9 & 5,3 & 35,6 & 7,7 & 82 & 124 \\
\hline & $20 / 5 / 98$ & $\mathrm{ME}$ & 4 & 141 & 6,8 & 2,6 & 44,5 & 13,3 & 68 & 235 \\
\hline & $27 / 5 / 98$ & A & 4 & 141 & 6,7 & 2,1 & 43,8 & 14,2 & 90 & 311 \\
\hline & $3 / 6 / 98$ & M & 5 & 141 & 6,6 & 2,5 & 56,1 & 21,8 & 76 & 412 \\
\hline & $10 / 6 / 98$ & $\mathrm{ME}$ & 3 & 142 & 6,7 & 3,3 & 48,3 & 15,9 & 80 & 303 \\
\hline & $17 / 6 / 98$ & A & 4 & 145 & 6,8 & 4,1 & 43,3 & 11,6 & 73 & 253 \\
\hline & $24 / 6 / 98$ & M & 4 & 151 & 6,8 & 4,6 & 41,8 & 10,8 & 74 & 127 \\
\hline
\end{tabular}


(cont.)

\begin{tabular}{|c|c|c|c|c|c|c|c|c|c|c|}
\hline & Semanas & Macrófita & $\begin{array}{c}\text { Turb } \\
\text { (NTU) }\end{array}$ & $\begin{array}{c}\text { Cond } \\
\left(\mu \mathrm{S} . \mathrm{cm}^{-1}\right)\end{array}$ & $\begin{array}{l}\mathrm{pH} \\
(\mathrm{pH})\end{array}$ & $\begin{array}{c}\mathrm{O}_{2} \\
\left(\mu \mathrm{g} \cdot \mathrm{L}^{-1}\right)\end{array}$ & $\begin{array}{c}\mathrm{CO}_{2} \mathrm{~T} \\
\left(\mu \mathrm{g} . \mathrm{L}^{-1}\right)\end{array}$ & $\begin{array}{c}\mathrm{CO}_{2} \mathrm{~L} \\
\left(\mu \mathrm{g} \cdot \mathrm{L}^{-1}\right)\end{array}$ & $\begin{array}{c}\text { PT } \\
\left(\mu \mathrm{g} \cdot \mathrm{L}^{-1}\right)\end{array}$ & $\begin{array}{c}\mathrm{NH}_{4} \\
\left(\mu \mathrm{g} . \mathrm{L}^{-1}\right)\end{array}$ \\
\hline \multirow{12}{*}{ 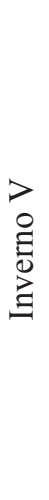 } & $1 / 7 / 98$ & M & 4 & 150 & 6,8 & 2,8 & 44,4 & 12,9 & 74 & 207 \\
\hline & 8/7/98 & M & 4 & 160 & 6,8 & 2,6 & 43,9 & 11,9 & 87 & 280 \\
\hline & $15 / 7 / 98$ & M & 4 & 159 & 6,7 & 4,0 & 46,0 & 14,6 & 54 & 207 \\
\hline & $22 / 7 / 98$ & M & 4 & 158 & 6,9 & 6,2 & 42,0 & 10,4 & 66 & 227 \\
\hline & 29/7/98 & A & 4 & 144 & 6,8 & 5,9 & 42,4 & 11,0 & 106 & 44 \\
\hline & $5 / 8 / 98$ & $\mathrm{ME}$ & 3 & 156 & 6,9 & 4,6 & 48,6 & 15,9 & 90 & 188 \\
\hline & $12 / 8 / 98$ & $\mathrm{ME}$ & 4 & 153 & 6,8 & 4,6 & 46,0 & 12,0 & 65 & 188 \\
\hline & $19 / 8 / 98$ & $\mathrm{ME}$ & 4 & 149 & 6,8 & 4,6 & 47,3 & 13,5 & 97 & 87 \\
\hline & $26 / 8 / 98$ & $\mathrm{ME}$ & 4 & 146 & 7,0 & 5,2 & 43,5 & 9,1 & 157 & 68 \\
\hline & $2 / 9 / 98$ & $\mathrm{ME}$ & 4 & 149 & 6,9 & 3,1 & 47,1 & 11,7 & 77 & 279 \\
\hline & 9/9/98 & A & 5 & 161 & 6,8 & 1,6 & 51,9 & 18,2 & 102 & 368 \\
\hline & $16 / 9 / 98$ & $\mathrm{ME}$ & 6 & 158 & 7,1 & 2,4 & 44,4 & 9,5 & 125 & 280 \\
\hline
\end{tabular}

Tabela 3. Coeficientes de correlação de Pearson e Kendall entre as variáveis físicas, químicas e biológicas da água e os dois primeiros eixos da ordenação pela PCA para o período de estudo $(\mathrm{N}=54)$.

Table 3. Pearson and Kendall correlation coefficients among the physical, chemical and biological variables related to water and the two first ordination axes of PCA along the period of study $(\mathrm{N}=54)$.

\begin{tabular}{|c|c|c|c|}
\hline \multirow[b]{2}{*}{ Variáveis } & \multirow[b]{2}{*}{ Abreviações } & \multicolumn{2}{|c|}{ Componentes Principais } \\
\hline & & Eixo 1 & Eixo 2 \\
\hline \multicolumn{4}{|c|}{ Ambientais } \\
\hline Temperatura & Temp & $-0,715$ & $-0,053$ \\
\hline Transparência & Transp & 0,705 & 0,352 \\
\hline Zona de Mistura & $Z_{\text {mix }}$ & 0,876 & 0,032 \\
\hline Macrófita & Macr & 0,607 & 0,017 \\
\hline Turbidez & Turb & $-0,745$ & $-0,288$ \\
\hline $\mathrm{pH}$ & $\mathrm{pH}$ & 0,088 & $-0,738$ \\
\hline Oxigênio Dissolvido & $\mathrm{O}_{2}$ & 0,256 & $-0,699$ \\
\hline Gás Carbonico Livre & $\mathrm{CO}_{2} \mathrm{~L}$ & $-0,298$ & 0,679 \\
\hline Fósforo Total & P-PT & $-0,517$ & $-0,197$ \\
\hline Amônio & $\mathrm{N}-\mathrm{NH}_{4}$ & $-0,265$ & 0,715 \\
\hline \multicolumn{4}{|c|}{ Biológicas } \\
\hline C. raciborskii & C.racib & 0,262 & 0,287 \\
\hline Clorofila $a$ & Cloro a & 0,483 & $-0,057$ \\
\hline Chlorophyceae & Chloro & $-0,591$ & 0,250 \\
\hline Cryptophyceae & Crypto & $-0,253$ & $-0,157$ \\
\hline Cyanophyceae & Cyano & 0,635 & 0,261 \\
\hline Riqueza & $\mathrm{R}$ & 0,013 & 0,188 \\
\hline Diversidade & $\mathrm{H}^{\prime}$ & 0,306 & $-0,346$ \\
\hline Uniformidade & U' & 0,317 & $-0,401$ \\
\hline Dominância & $D^{\prime}$ & $-0,401$ & 0,312 \\
\hline \multicolumn{2}{|c|}{ Total de Explicabilidade: } & $36,39 \%$ & $18,92 \%$ \\
\hline
\end{tabular}

Sant'Anna, Microcystis aeruginosa (Kützing) Kützing, Merismopedia trollerii Bachmann, Merismopedia glauca (Ehrenberg) Kützing, Synechococcus nidulans (Pringsheim) Komárek, Synechocystis aquatilis Sauvageau, Pseudanabaena mucicula (HuberPestalozzi \& Naumann) Schwabe, Planktothrix agardhii (Gomont) Komárek \& Anagnostidis), além de Closterium gracile Brébisson ex Ralfs (Chlorophyta). Branco \& Cavalcanti (1999), Giani \& Pinto-Coelho (1986) e Branco \& Senna (1996b) também registraram riqueza de táxons fitoplanctônicos relativamente elevada, no lago Paranoá, junto com florações de C. raciborskii.

Estes resultados podem ser explicados pelas diferenças entre florações de Microcystis e de Cylindrospermopsis. Quando a floração é de espécies de Microcystis, suas colônias com ampla mucilagem proporcionam grande sombreamento para as demais espécies fitoplanctônicas, dificultando seu desenvolvimento. Como consequência, a diversidade e riqueza diminuem significativamente (Ramirez 1996). No presente caso, a floração de Cylindrospermopsis (curta e não muito intensa), que possui tricomas finos e alongados, não promoveu tal sombreamento, permitindo a coexistência de outras espécies.

Comparando o período de primavera e verão de 1997 com o mesmo período de 1994 e 1995, quando foram registradas florações de Microcystis aeruginosa (Kützing) Kützing (Ramirez 1996, Nogueira 1997), diferentes resultados, em relação à espécie dominante, foram verificados. Os fatores discutidos detalhadamente por Nogueira (1997), para explicar a floração de M. aeruginosa, foram estabilidade térmica do sistema, 
Tabela 4. Síntese dos resultados da CCA baseada em seis variáveis ambientais e nove variáveis biológicas $(\mathrm{N}=54)$.

Table 4. Synthesis of the results of the CCA based on six environmental and nine biological variables $(\mathrm{N}=54)$.

\begin{tabular}{lcc}
\hline & Eixo 1 & Eixo 2 \\
\hline Autovalores $(\lambda)$ & 0,104 & 0,042 \\
Porcentagem de Variância explicada (\%) & 18,6 & 7,5 \\
Porcentagem de variância acumulada & 18,6 & 26,1 \\
Correlação de Pearson (espécie-ambiente) & 0,783 & 0,494 \\
Teste de Monte Carlo (p) & 0,040 & 0,020 \\
$\quad$ Autovalores & 0,030 & 0,040 \\
\hline
\end{tabular}

capacidade de flutuação das colônias de Microcystis, baixa razão NT/PT, altos valores de $\mathrm{pH}$ e baixos valores de $\mathrm{CO}_{2}$. Com exceção da razão NT/PT, não avaliada no presente trabalho, as demais condições que favoreceram o desenvolvimento de Microcystis foram semelhantes às atuais. Assim, pergunta-se por que a floração na primavera de 1997 foi de C. raciborskii e não de $M$. aeruginosa como vinha ocorrendo em anos anteriores? A resposta parece estar diretamente relacionada à redução nas concentrações de PT provocada pela intensa absorção desse nutriente pelas macrófitas, levando à substituição de Microcystis aeruginosa por Cylindrospermopsis raciborskii. Esta última espécie, conforme dados de literatura (Jensen et al. 1994, Padisák 1997) tem capacidade de se desenvolver em concentrações de fósforo já limitantes para outras cianobactérias, o que certamente impediu o rápido desenvolvimento de $M$. aeruginosa e favoreceu a dominância de $C$. raciborskii.

Agradecimentos - Aos colegas Cynthia B. Fürstenberg, Ilka S. Vercellino, Maria Auxiliadora e Clóvis F. do Carmo (IBt) pelo auxílio nas coletas e análises físicas e químicas da água. Ao CNPq pela bolsa de doutorado (processo 142.841/19972001) concedida à primeira autora e pela bolsa de produtividade em pesquisa (processo 30030016/81) concedida à segunda autora. Esta pesquisa faz parte do Projeto Tipologia, Monitoramento e Recuperação de Corpos d'água da Reserva Biológica do Parque Estadual das Fontes do Ipiranga, São Paulo financiado pelo $\mathrm{CNPq}$ (processo 520745/96-5).

Tabela 5. Coeficientes canônicos e correlações "intra-set" das seis variáveis ambientais com os eixos 1 e 2 da CCA, baseada nas nove variáveis biológicas, no período de estudo $(\mathrm{N}=54)$.

Table 5. Canonical coefficients and intra-set correlations from six environmental variables with the CCA axis 1 and 2, based on nine biological variables, along the period of study $(\mathrm{N}=54)$.

\begin{tabular}{llcrrr}
\hline & \multicolumn{2}{c}{ Coeficiente Canônico } & & \multicolumn{2}{c}{$\begin{array}{c}\text { Coeficiente de Correlação } \\
\text { "intra-set" }\end{array}$} \\
\cline { 2 - 3 } Variável & Eixo 1 & Eixo 2 & & Eixo 1 & Eixo 2 \\
\hline Transparência (transp) & $-0,984$ & $-0,006$ & & $-0,961$ & $-0,071$ \\
Zona de Mistura (Zmix) & $-0,303$ & 0,654 & & $-0,543$ & 0,506 \\
Fósforo Total (P-PT) & $-0,307$ & $-0,016$ & 0,255 & $-0,113$ \\
Amônio (N-NH $\left.{ }_{4}\right)$ & $-0,004$ & $-0,572$ & & 0,009 & $-0,509$ \\
pH & 0,100 & 1,351 & 0,226 & 0,599 \\
Gás Carbônico Total $\left(\mathrm{CO}_{2} \mathrm{~T}\right)$ & 0,038 & 1,190 & & 0,044 & $-0,346$ \\
\hline
\end{tabular}


Tabela 6. Valor médio, máximo, mínimo, desvio padrão (DP) e coeficiente de variação de Pearson = CV (\%) para as quatro estações do ano e para a variação anual da densidade total $=\mathrm{DT}\left(\right.$ org. $\left.\mathrm{mL}^{-1}\right)$, riqueza $=\mathrm{R}$, diversidade $=\mathrm{H}^{\prime}$, uniformidade $=\mathrm{U}^{\text {, }}$ e dominância $=\mathrm{D}^{\prime}$.

Table 6. Medium, maximum and minimum values, standard deviation (DP) and Pearson variation coefficient $=\mathrm{CV}(\%)$ of the four seasons and for anual variation of total density $=\mathrm{DT}\left(\right.$ org. $\left.\mathrm{mL}^{-1}\right)$, richness $=\mathrm{R}$, diversity $=\mathrm{H}^{\prime}$, unifomity $=\mathrm{U}^{\prime}$, and dominance $=\mathrm{D}^{\prime}$.

\begin{tabular}{|c|c|c|c|c|c|c|}
\hline & & DT & $\mathrm{R}$ & $\mathrm{H}^{\prime}$ & U' & $D^{\prime}$ \\
\hline \multirow{5}{*}{ 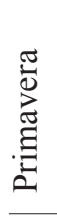 } & Média & 28.284 & 58 & 4,20 & 0,72 & 0,1264 \\
\hline & Máximo & 40.706 & 79 & 4,95 & 0,83 & 0,3222 \\
\hline & Mínimo & 9.428 & 30 & 2,98 & 0,53 & 0,0555 \\
\hline & DP & 10.537 & 12 & 0,58 & 0,09 & 0,0799 \\
\hline & $\mathrm{CV}(\%)$ & 37 & 21 & 14 & 12 & 63 \\
\hline \multirow{5}{*}{ 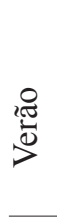 } & Média & 24.164 & 54 & 4,43 & 0,77 & 0,1178 \\
\hline & Máximo & 39.674 & 65 & 5,21 & 0,89 & 0,4659 \\
\hline & Mínimo & 13.867 & 39 & 2,36 & 0,45 & 0,0364 \\
\hline & DP & 7.080 & 8 & 0,89 & 0,14 & 0,1329 \\
\hline & $\mathrm{CV}(\%)$ & 29 & 14 & 20 & 18 & 113 \\
\hline \multirow{5}{*}{$\begin{array}{l}\text { : } \\
\stackrel{0}{0} \\
0\end{array}$} & Média & 26.221 & 63 & 4,79 & 0,80 & 0,0686 \\
\hline & Máximo & 56.102 & 80 & 5,25 & 0,86 & 0,1485 \\
\hline & Mínimo & 14.366 & 46 & 3,98 & 0,72 & 0,0404 \\
\hline & $\mathrm{DP}$ & 11.324 & 11 & 0,38 & 0,05 & 0,0356 \\
\hline & $\mathrm{CV}(\%)$ & 43 & 17 & 8 & 6 & 52 \\
\hline \multirow{5}{*}{$\begin{array}{l}\stackrel{\Xi}{\Xi} \\
\stackrel{0}{\Xi}\end{array}$} & Média & 48.396 & 61 & 3,31 & 0,56 & 0,3010 \\
\hline & Máximo & 90.824 & 77 & 4,87 & 0,84 & 0,6306 \\
\hline & Mínimo & 26.733 & 48 & 1,75 & 0,29 & 0,0499 \\
\hline & DP & 16.112 & 10 & 0,83 & 0,14 & 0,1533 \\
\hline & $\mathrm{CV}(\%)$ & 33 & 16 & 25 & 25 & 51 \\
\hline \multirow{5}{*}{ 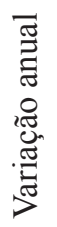 } & Média & 32.027 & 59 & 4,13 & 0,70 & 0,1595 \\
\hline & Máximo & 90.824 & 80 & 5,25 & 0,89 & 0,6306 \\
\hline & Mínimo & 9.428 & 30 & 1,75 & 0,29 & 0,0364 \\
\hline & DP & 14,8 & 10 & 0,88 & 0,14 & 0,14 \\
\hline & $\mathrm{CV}(\%)$ & 46 & 17 & 21 & 20 & 87 \\
\hline
\end{tabular}

\section{Referências bibliográficas}

ALVES, V.R.E., CAVALCANTI, C.G.B. \& MATTOS, S.P. 1988. Análise comparativa de parâmetros físicos, químicos e biológicos em um período de 24 horas no Lago Paranoá, Brasília, DF, Brasil. Acta Limnologica Brasiliensia 2:199-218.

BEYRUTH, Z. 1992. Macrófitas aquáticas de um lago marginal ao rio Embú-Mirim, São Paulo, Brasil. Revista Saúde Pública 26:272-282.

BEYRUTH, Z. 1994. Eichhornia crassipes (Mart.) Solms. as pollution indicator. Verhandlungen der Internatonalen Vereinigung fur Theorestische und Angewandte Limnologie 545:1-4.

BICUDO, C.E.M., RAMIREZ, J.J.R., TUCCI-MOURA, A.N. \& BICUDO, D.C. 1999. Dinâmica de populações fitoplanctônicas em ambiente eutrofizado: o Lago das Garças, São Paulo. In Ecologia de reservatórios: estrutura, função e aspectos sociais. (R. Henry, ed.), Fapesp/Fundibio, Botucatu, p.449-508.
BICUDO, D.C., TUCCI-MOURA, A.N., RAMIREZ, J.J.R., CARMO, C.F., NOGUEIRA, N.M.C. \& BICUDO, C.E.M. 1999. Escala de amostragem e variabilidade de fatores limnológicos em reservatório eutrofizado (Lago das Garças, São Paulo). In Ecologia de reservatórios: estrutura, função e aspectos sociais. (R. Henry, ed.), Fapesp/Fundibio, Botucatu, p.409-448.

BOUVY, M., FALCÃO, D., MARINHO, M., PAGANO, M. \& MOURA, A. 2000. Occurrence of Cylindrospermopsis (Cyanobacteria) in 39 Brazilian tropical reservoirs during the 1998 drought. Aquatic Microbial Ecology 23:13-27.

BOUVY, M., MOLICA, R., OLIVEIRA, S., MARINHO, M. \& BEKER, B. 1999. Dynamics of a toxic cyanobacterial bloom (Cylindrospermopsis raciborskii) in a shallow reservoir in the semi-arid region of northeast Brazil. Aquatic Microbial Ecology 20:285-297. 
BRANCO, C.W.C. \& CAVALCANTI, C.G.B. 1999. A ecologia das Comunidades Planctônicas no Lago Paranoá. In Ecologia de reservatórios: estrutura, função e aspectos sociais. (R. Henry, ed.). Fapesp/Fundibio, Botucatu, p.573-595.

BRANCO, C.W.C. \& SENNA, P.A.C. 1991. The taxonomic elucidation of the Paranoá Lake (Brasilia, Brazil) problem: Cylindrospermopsis raciborskii. Bulletin du Jardin Botanique National de Belgique 61:85-91.

BRANCO, C.W.C.\& SENNA, P.A.C. 1994. Factors influencing the development of Cylindrospermopsis raciborskii and Microcystis aeruginosa in Paranoá Reservoir, Brasília, Brazil. Algological Studies 75:85-96.

BRANCO, C.W.C. \& SENNA, P.A.C. 1996a. Relations among heterotrophic bacteria, chlorophyll $a$, total phytoplankton, total zooplankton and physical and chemical features in the Paranoá reservoir, Brasília, Brazil. Hydrobiologia 337:171-181.

BRANCO, C.W.C. \& SENNA, P.A.C. 1996b. Phytoplankton composition, community structure and seasonal changes in a tropical reservoir (Paranoá Reservoir, Brazil). Algological Studies 81:69-84.

CARMICHAEL, W.W. 1996. Toxic Microcystis and the environment. In Toxic Microcystis (M.F. Watanabe, K.I. Harada, W.W. Carmichael \& H. Fujiki, eds.). CRC Press, Boca Raton, p.1-11.

CARMO, C.F. 2000. Aporte de nutrientes, nitrogênio e fósforo, e sua relação com os impactos antropogênicos em um lago urbano, São Paulo, SP, Brasil. Dissertação de mestrado, Universidade de São Paulo, São Carlos.

CODD, G.A. 2000. Cyanobacterial toxins, the perception of water quality, and the prioritisation of eutrophication control. Ecological Engineering 16:51-60.

COLE, G. 1979. Textbook of limnology. $2^{\text {nd }}$ ed. C.V. Mosby, Saint Louis.

COLE, G. 1994. Textbook of limnology. $4^{\text {th }}$ ed. Waveland Press, Illinois.

FULTON, R.S. \& PAERL, H.W. 1987. Effects of colonial morphology on zooplankton utilization of algal resouces during blue-green algal (Microcystis aeruginosa) blooms. Limnology \& Oceanography 32:634-644.

GENTIL, R.C. 2000. Variação sazonal do fitoplâncton de um lago subtropical eutrófico e aspectos sanitários, São Paulo, SP. Dissertação de mestrado, Faculdade de Saúde Pública da Universidade de São Paulo, São Paulo.

GIANI, A. \& PINTO-COELHO, R.M. 1986. Contribuição ao conhecimento das algas fitoplanctônicas do reservatório do Paranoá, Brasília, Brasil: Chlorophyta, Euglenophyta, Pirrophyta e Schizophyta. Revista Brasileira de Botânica 9:45-62.

GOLTERMAN, H.L., CLYMO, R.S. \& OHSTAD, M.A.M. 1978. Methods for physical and chemical analysis of freshwater. Blackwell Scientific Publications, Oxford.
HORECKÁ, M. \& KOMÁREK, J. 1979. Taxonomic position of three planktonic blue-green algae from the genera Aphanizomenon and Cylindrospermopsis. Preslia 51:289-312.

HUSZAR, V.L.M., SILVA, L.H.S., MARINHO, M.M., DOMINGOS, P. \& SANT'ANNA, C.L. 2000. Cyanoprokaryote assemblages in eight productive tropical Brazilian waters. In The Trophic Spectrum Revisited: The Influence of Trophic State on the Assembly of Phytoplankton Communities (C.S. Reynolds, M.Dokulil \& J. Padisák, eds.). Kluwer Academic Publishers, Dordrecht, p.67-77.

JEEJI-BAI, N., HEGEWALD, E. \& SOEDER, C.J. 1977. Revision and taxonomic analysis of genus Anabaenopsis. Algological Studies 18:25-32.

JENSEN, J.P., JEPPESEN, E., OLRIK, K. \& KRISTENSEN, P. 1994. Impact of nutrients and physical factors on the shift from cyanobacterial to chlorophyte dominance in shallow Danish lakes. Canadian Journal of Fisheries and Aquatic Sciences 51:1692-1699.

KOMÁRKOVÁ, J. 1998. The tropical planktonic genus Cylindrospermopsis (Cyanophytes, Cyanobacteria). In Anais do IV Congresso Latino-Americano de Ficologia (M.T.P. Azevedo, D.P. Santos, L.S.C. Pinto, M. Menezes, M.T. Fujii, N.S. Yokoya, P.A.C. Senna \& S.M.P.B. Guimarães, eds.). Sociedade Ficológica da America Latina e Caribe, São Paulo. v.1, p.327-340.

KOMÁRKOVÁ, J., LAUDARES-SILVA, R. \& SENNA, P.A.C. 1999. Extreme morphology of Cylindrospermopsis raciborskii (Nostocales, Cyanobacteria) in the Lagoa do Peri, a freshwater coastal lagoon, Santa Catarina, Brazil. Algological Studies 94:207-222.

LAGOS, N., ONODERA, H., ZAGATTO, P.A., ANDRINOLO, D., AZEVEDO, S.M.F.Q. \& OSHIMA, Y. 1999. The first evidence of paralytic shellfish toxins in the freshwater cyanobacterium Cylindrospermopsis raciborskii, isolated from Brazil. Toxicon 37:1359-1373.

LLOYD, M. \& GHELARDI, R.J. 1964. A table for calculating the equitability component of species diversity. Journal of Ecology 33:217-225.

LUND, J.W.G., KIPLING, C. \& LECREN, E.D. 1958. The invert microscope method of estimating algal numbers and the statistical basis of estimations by counting. Hydrobiologia 11:143-170.

MERCANTE, C.T. \& TUCCI, A. 1999. Comparação entre os índices de Carlson e de Carlson modificado aplicados a dois ambientes aquáticos subtropicais, São Paulo, SP. Acta Limnologica Brasiliensia 11:1-14.

MOURA, A.T.N. 1996. Estrutura e dinâmica da comunidade fitoplanctônica numa lagoa eutrófica, São Paulo, SP, Brasil, a curtos intervalos de tempo: comparação entre épocas de chuva e seca. Dissertação de mestrado, Universidade Estadual Paulista, Rio Claro. 
NOGUEIRA, N.M.C. 1997. Dinâmica populacional de Microcystis aeruginosa Kutzing (Cyanophyceae/ Cyanobacteria) ao longo de um ano no Lago das Garças, São Paulo, SP, Brasil. Dissertação de mestrado, Universidade Estadual Paulista, Rio Claro.

NOGUEIRA, N.M.C. \& RAMIREZ, J.J.R. 1998. Variação mensal da condição trófica do Lago das Garças (São Paulo, SP, Brasil). Acta Limnologica Brasiliensia 10:21-34.

OHTANI, I., MOORE, R.E. \& RUNNEGAR, M.T.C. 1992. Cylindrospermopsin: a potent hepatotoxin from the bluegreen alga Cylindrospermopsis raciborskii. Journal of the American Chemical Society 114:7941-7942.

PADISÁK, J. 1997. Cylindrospermopsis raciborskii (Woloszynnska) Seenayya et Subba Raju, an expanding, highly adaptive cyanobacterium: worldwide distribution and review of its ecology. Archiv für Hydrobiology 107:563-593.

PALMER, C.M. 1969. Report on the algae in relation to water quality of Paranoa Lake, Brasilia: Relatório de consultoria. Pan American Health Organization/WHO, Brasília.

PINTO-COELHO, R.M. 1983. Efeitos do zooplâncton na composição qualitativa e quantitativa do fitoplâncton no Lago Paranoá, Brasília, DF, Brasil. Dissertação de mestrado, Universidade de Brasília, Brasília.

PINTO-COELHO, R.M., COELHO, M.M., ESPÍRITOSANTO, M.M. \& CORNELISSEN, T.G. 1999. Efeitos da eutrofização na estrutura da comunidade planctônica na Lagoa da Pampulha, Belo Horizonte, MG. In Ecologia de reservatórios: estrutura, função e aspectos sociais. (R. Henry, ed.), Fapesp/Fundibio, Botucatu, p.551-572.

RAMÍREZ, J.J.R. 1996. Variações espacial vertical e nictemeral da estrutura da comunidade fitoplanctônica e variáveis ambientais em quatro dias de amostragens de diferentes épocas do ano no Lago das Garças, São Paulo. Tese de doutorado, Universidade de São Paulo, São Paulo.

REYNOLDS, C.S. 1984. Phytoplankton periodicity: the interations of form, function and environmental variability. Freshwater Biology 14:111-142.

SAKER, M.L. \& NEILAN, B.A. 2001. Varied diazotrophies, morphologies, and toxicities of genetically similar isolates of Cylindrospermopsis raciborskii (Nostocales, Cyanophyceae) from Northern Australia. Applied and Environmental Microbiology 67:1839-1845.

SANT'ANNA, C.L. \& AZEVEDO, M.T.P. 2000. Contribution to the knowledge of potentially toxic Cyanobacteria from Brazil. Nova Hedwigia 71:359-385.
SANT’ANNA, C.L., AZEVEDO, M.T.P. \& SORMUS, L. 1989. Fitoplâncton do Lago das Garças, Parque Estadual das Fontes do Ipiranga, São Paulo, SP, Brasil: estudo taxonômico e aspectos ecológicos. Hoehnea 16:89-131.

SANT'ANNA, C.L., SORMUS, L., TUCCI, A. \& AZEVEDO, M.T.P. 1997. Variação sazonal do fitoplâncton do Lago das Garças, São Paulo, SP. Hoehnea 24:67-86.

SANT'ANNA, C.L., XAVIER, M.B. \& SORMUS, L. 1988. Estudo qualitativo do fitoplâncton da represa de Serraria, Estado de São Paulo, Brasil. Revista Brasileira de Biologia 48:83-102.

SARTORY, D.P. \& GROBBELAAR, J.U. 1984. Extraction of chlorophyll $a$ from freshwater phytoplankton for spectrophotometric analysis. Hidrobiologia 114:177-187.

SHANNON, C.E. \& WEAVER, W. 1963. The mathematical theory of communication. University of Illinois Press, Urbana.

SHEPHERD, G.J. 1996. Fitopac 1: Manual de usuário. Departamento de Botânica, Unicamp, Campinas.

SIMPSON, E.H. 1949. Measurement of diversity. Nature 163:688.

SOLORZANO, L. 1969. Determination of amonia in natural waters by the phenolhypoclorite method. Limnology \& Oceanography 14:799-801.

SOUZA, R.C.R., CARVALHO, M.C.\& TRUZZI, A.C. 1998. Cylindrospermopsis raciborskii (Wolosz.) Seenaya and a contribution to the knowledge of Rio Pequeno arm, Billings Reservoir, Brazil. Environmental Toxicology and Water Quality 13:73-81.

STARLING, F.L.R.M. 1989. Estudo experimental dos impactos de peixes planctófagos sobre a comunidade planctônica e a qualidade da água do Lago Paranoá, Brasília, DF. Dissertação de mestrado, Universidade de Brasília, Brasília.

TORGAN, L.C. \& GARCIA, M. 1989. Novas ocorrências (Cyanophyta e Chlorophyta) para a ficoflora planctônica do Rio Grande do Sul. Hoehnea 16:57-64.

UTERMÖHL, H. 1958. Zur Vervolkomnung der quantitativen Phytoplankton: methodik. Mitteilungen Internationale Vereinigung fur Theoretische und Angewandte Limnologie 9:1-38.

VALDERRAMA, J.C. 1981. The simultaneous analysis of total nitrogen and total phosphorus in natural waters. Marine Chemistry 10:109-122.

WETZEL, R.G. 1981. Limnologia. Omega, Barcelona.

WETZEL, R.G. \& LIKENS, G.E. 1991. Limnological analysis. Springer Verlag, New York. 